\title{
ASHA GARG
}

\section{Heterogeneous questionnaires and Rényi's entropy}

Revue française d'automatique, d'informatique et de recherche opérationnelle. Recherche opérationnelle, tome 15, no 4 (1981), p. 389-395.

<http://www.numdam.org/item?id=RO_1981_15_4_389_0>

(C) AFCET, 1981, tous droits réservés.

L'accès aux archives de la revue « Revue française d'automatique, d'informatique et de recherche opérationnelle. Recherche opérationnelle » implique l'accord avec les conditions générales d'utilisation (http://www.numdam.org/ legal.php). Toute utilisation commerciale ou impression systématique est constitutive d'une infraction pénale. Toute copie ou impression de ce fichier doit contenir la présente mention de copyright.

\section{Numdam}

Article numérisé dans le cadre du programme

Numérisation de documents anciens mathématiques

http://www.numdam.org/ 


\title{
HETEROGENEOUS QUESTIONNAIRES AND RÉNYI'S ENTROPY ( $\left.{ }^{*}\right)$
}

\author{
by Asha GARG $\left({ }^{1}\right),\left({ }^{2}\right)$
}

\begin{abstract}
Recently we considered a heterogeneous questionnaire and characterized an average charge of order $t$ by using the additivity property. We found that it is bounded from below by Rényi's Entropy of order $\alpha$, where $\alpha=1 /(1+t)$.

In this paper we have extended this work for the more general case when the order of both, the average charge and the bound, is same i.e. equal to $\alpha$. This gives a new measure of average charge. We have characterized it and a related coding theorem has also been established.

Résumé. - Nouts atons récemment considéré un questionnaire hétérogène et caractérisé la charge moyenne d'ordre $t$ en utilisant la propriété d'additivité. Nous avons trouvé qu'elle est bornée inférieurement par l'entropie de Rényi d'ordre $\alpha=1 /(1+t)$.

Dans cet article nous étendrons le travail au cas plus général où les ordres de la charge moyenne et de la borne sont les mêmes, c'est-à-dire égaux à $\alpha$. Cela donne une nouvelle mesure de la charge moyenne. Nous en donnons une caractérisation, et nous établissons un théorème de codage.
\end{abstract}

\section{INTRODUCTION}

Recently, Duncan [2] considered heterogeneous questionnaires and found that if $\log _{2} d$ is considered as the charge for a question of resolution $d$ then the expected charge for the questionnaire is lower bounded by Shannon entropy. For an arbitrary heterogeneous questionnaire Sharma and Garg [5] defined a measure of average charge of order $t$ as:

$$
E_{P}^{t} C(Q)=\frac{1}{t} \log _{2}\left[\sum_{i=1}^{m} p_{i} \prod_{d=1}^{\infty} d^{t n_{i d}}\right]
$$

$\left({ }^{*}\right)$ Received May 1980.

( ${ }^{1}$ ) Department of Mathematics, University of Delhi, India.

$\left({ }^{2}\right)$ This work has been performed during a research fellowship from the Council of Scientific and Industrial Research, New Delhi, India. The financial help is gratefully acknowledged.

R.A.I.R.O. Recherche opérationnelle/Operations Research, 0399-0559/1981/389/\$5.00

(C) AFCET-Bordas-Dunod 
where $n_{i d}$ represents the number of questions of resolution $d$ required to determine the $i$-th element of the state space $\Theta=\left\{\theta_{1}, \theta_{2}, \ldots, \theta_{m}\right\}$ and $P=\left\{p_{1}, p_{2}, \ldots, p_{m}\right\}$ is the probability distribution associated with $\Theta$ such that:

$$
\sum_{i=1}^{m} p_{i}=1 ; \quad p_{i} \geqq 0 \quad(i=1,2, \ldots, m) .
$$

We then found that if $\alpha=1 /(1+t)$, the average charge defined by equation ( 1$)$ is bounded from below by Rényi's entropy of order $\alpha$ [4] with equality iff:

$$
\prod_{d=2}^{m} d^{-n_{i d}}=\frac{p_{i}^{\alpha}}{\sum_{j=1}^{m} p_{j}^{\alpha}} \quad \text { and } \quad n_{i d}=0, \quad \forall d>m .
$$

We have extended this work for a more general case when the measure of average charge and the bound are of the same order, $\alpha$. The condition of equality, which holds for this case is:

$$
p_{i}=\prod_{d=2}^{m} d^{-n_{i d} ;} \quad n_{i d}=0 \quad \text { for all } d>m .
$$

This condition is the same as that obtained by Duncan [2] for the inequality:

$$
\mathrm{E}_{\mathrm{p}} \mathrm{C}(\mathrm{Q}) \geqq H(P),
$$

where $E_{P} C(Q)$ is the expected charge defined by Duncan [2] for a heterogeneous questionnaire and $H(P)$ is the Shannon's entropy. We have obtained a new measure of average charge and have established a related coding theorem.

II. Since we are interested in the more general case when the order of average charge and the bound is same, we have to modify the definition of average charge given in equation (1). Let us define the average charge as the most general quasilinear mean-value, i.e.:

$$
E_{P}^{(J . \varphi)} C(Q)=\varphi^{-1} \frac{\sum_{i=1}^{m} f\left(p_{i}\right) \varphi\left(\log _{2} \prod_{d=1}^{\infty} d^{n_{i d}}\right)}{\sum_{i=1}^{m} f\left(p_{i}\right)}
$$

where $f$ is a non-constant positive-valued continuous function defined on $(0,1)$, $\varphi$ is a strictly monotonically increasing and continuous real-valued function defined on $\left[1, \infty\left[\right.\right.$ and $\varphi^{-1}$ denotes the inverse function of $\varphi$.

Every measure of average charge is required to satisfy the condition that whenever $n_{i d}(i=1,2, \ldots, m)$ increases by " $m_{d}$ " $\left(m_{d}\right.$ is $a+v e$ integer, 
$d=1, \ldots, \infty)$, it should also increase by $\sum_{d=1}^{\infty} m_{d} \log _{2} d$. Thus, we get the functional equation as:

$$
\begin{gathered}
\varphi^{-1}\left[\frac{\sum_{i=1}^{m} f\left(p_{i}\right) \varphi\left(\log _{2} \prod_{d=1}^{\infty} d^{n_{i d}}+\sum_{d=1}^{\alpha} \log _{2} d^{m_{d}}\right)}{\sum_{i=1}^{m} f\left(p_{i}\right)}\right] \\
=\varphi^{-1}\left[\frac{\sum_{i=1}^{m} f\left(p_{i}\right) \varphi\left(\log _{2} \prod_{d=1}^{\infty} d^{n_{i d}}\right)}{\sum_{i=1}^{m} f\left(p_{i}\right)}\right]+\sum_{d=1}^{\infty} \log _{2} d^{m_{d}}
\end{gathered}
$$

This equation is the translativity equation and the following theorem can be easily established (Aczél [1]).

THEOREM 1: The only quasi-linear measures $E_{P}^{(f, \varphi)} C(Q)$ of average charge which are translative $(6)$ are $E_{P}^{\left(f, \varphi_{1}\right)} C(Q)$ and $E_{P}^{\left(t, \varphi_{a}^{\prime}\right)} C(Q)$ where:

$$
\begin{aligned}
\varphi_{1}(x)=b x+a, & b>0, \\
\varphi_{\alpha}(x)=b_{2}^{(\alpha-1) x}+a, & b(\alpha-1)>0
\end{aligned}
$$

and:

$$
\begin{gathered}
E_{P}^{\left(f, \varphi_{1}\right)} C(Q)=\frac{\sum_{i=1}^{m} f\left(p_{i}\right) \log _{2} \prod_{d=1}^{\infty} d^{n_{i d}}}{\sum_{i=1}^{m} f\left(p_{i}\right)}, \\
E_{P}^{\left(f, \varphi_{a}\right)} C(Q)=\frac{1}{\alpha-1} \log _{2}\left[\frac{\sum_{i=1}^{m} f\left(p_{i}\right) \prod_{d=1}^{\infty} d^{(\alpha-1) n_{i d}}}{\sum_{i=1}^{m} f\left(p_{i}\right)}\right] ; \quad \alpha \neq 1 .
\end{gathered}
$$

To find a relation between Rényi's entropy and $\mathrm{E}_{\mathrm{P}}^{\left(f, \varphi_{a}\right)} C(Q)$, we rewrite equation (9) as:

$$
\begin{aligned}
E_{P}^{\left(f, \varphi_{\alpha}\right)} C(Q)=\frac{1}{\alpha-1} \log _{2}\left[\sum_{i=1}^{m} f\left(p_{i}\right)\right. & \left.\prod_{d=1}^{\infty} d^{(\alpha-1) n_{i d}}\right] \\
& +\frac{1}{1-\alpha} \log _{2}\left[\sum_{i=1}^{m} f\left(p_{i}\right)\right] ; \quad \alpha \neq 1 .
\end{aligned}
$$

vol. $15, \mathrm{n}^{\circ} 3$, novembre 1981 
We would restrict to $\alpha>0$ and select $f$ in such a way that the second term on the right hand side of equation (10) approaches Rényi's entropy, that is:

$$
\frac{1}{1-\alpha} \log _{2}\left[\sum_{i=1}^{m} f\left(p_{i}\right)\right]=\frac{1}{1-\alpha} \log _{2}\left[\sum_{i=1}^{m} p_{i}^{\alpha}\right] ; \alpha>0, \quad \alpha \neq 1
$$

Thus, from equation (11), we get the functional equation:

$$
\sum_{i=1}^{m} f\left(p_{i}\right)=\sum_{i=1}^{m} p_{i}^{\alpha} ; \quad \alpha>0, \quad \alpha \neq 1 .
$$

The only non-constant continuous solutions of (12) are of the form:

$$
f(p)=p^{\alpha} ; \quad \alpha>0, \quad \alpha \neq 1 .
$$

Hence:

$$
\begin{gathered}
E_{P}^{\left(f, \varphi_{\alpha}\right)} C(Q)=\frac{1}{\alpha-1} \log _{2}\left[\frac{\sum_{i=1}^{m} p_{i}^{\alpha} \prod_{d=1}^{\infty} d^{(\alpha-1) n_{i d}}}{\sum_{i=1}^{m} p_{i}^{\alpha}}\right]=E_{P}^{\alpha} C(Q) ; \\
\alpha>0, \quad \alpha \neq 1 .
\end{gathered}
$$

$E_{P}^{\alpha} C(Q)$ is an average charge of order $\alpha$. We can easily see that $\lim E_{P}^{\alpha} C(Q)=E_{P} C(Q)$. So, $E_{P} C(Q)$ can be considered as the average charge of $\alpha \rightarrow 1$ order 1 .

III. In this section we have obtained a relation between $E_{P}^{\alpha} C(Q)$ and Rényi's entropy.

THEOREM 3.1: If $n_{\text {id }}(i=1,2, \ldots, m)$ satisfy the condition:

$$
\sum_{i=1}^{m} \prod_{d=1}^{\infty} d^{-n_{i d}} \leqq 1
$$

then we must have:

$$
E_{P}^{\alpha} C(Q) \geqq H_{\alpha}(P),
$$

where:

$$
H_{\alpha}(P)=\frac{1}{1-\alpha} \log _{2}\left[\sum_{i=1}^{m} \mathrm{p}_{i}^{\alpha}\right] ; \quad \alpha \neq 1
$$

is the Rényi's entropy. 
In (16) equality would hold iff $n_{i d}=0$ for all $d>m$ and:

$$
p_{i}=\prod_{d=2}^{m} d^{-n_{i d}} \quad(i=1,2, \ldots, m) .
$$

Proof: Nath [3] has shown that:

$$
H_{\alpha}(P) \leqq H_{\alpha}\left(R_{\alpha}(P, Q) \| Q\right) ; \quad \alpha>0
$$

with equality iff $p_{i}=q_{i}, \forall i=1,2, \ldots, m$.

Substituting:

in (18), we get:

$$
q_{i}=\frac{\prod_{d=1}^{\infty} d^{-n_{i d}}}{\sum_{j=1}^{m} \prod_{d=1}^{\infty} d^{-n_{i d}}}
$$

$$
\begin{aligned}
& H_{\alpha}(P) \leqq \frac{1}{1-\alpha} \log _{2}\left[\sum_{i=1}^{m} p_{i}^{\alpha}\right] \\
&+\frac{1}{\alpha-1} \log _{2}\left[\frac{\sum_{i=1}^{m} p_{i}^{\alpha} \prod_{d=1}^{\infty} d^{(\alpha-1) n_{i d}}}{\left(\sum_{j=1}^{m} \prod_{d=1}^{\infty} d^{-n_{j d}}\right)^{1-\alpha}}\right] \\
& \leqq \frac{1}{1-\alpha} \log _{2}\left[\frac{\sum_{i=1}^{m} p_{i}^{\alpha} \prod_{d=1}^{\infty} d^{(\alpha-1) n_{i d}}}{\sum_{i=1}^{m} p_{i}^{\alpha}}\right] \text { (using [15]) } \\
&=E_{P}^{\alpha} C(Q) .
\end{aligned}
$$

Clearly, equality holds iff the conditions (17) are satisfied (see Duncan [2]). IV. In this section we have proved an analogous of noiseless coding theorem related to the measure $E_{P}^{\alpha} C(Q)$ of average charge.

From information theory we know that there always exists a uniquely decipherable binary code whose average length is bounded from above by Shannon entropy plus 1 . In questionnaire theory this implies that there always exists a homogeneous questionnaire of resolution 2 whose average charge also has the same upper bound. This suggests that (cf. Duncan [2]) there exists a heterogeneous questionnaire with the same upper bound,i.e.:

$$
\inf _{Q} E_{P} C(Q)<H(P)+1
$$


THEOREM 4.1: Let there be a state space $\Theta=\left\{\theta_{1}, \theta_{2}, \ldots, \theta_{m}\right\}$ with probability distribution $P=\left(p_{1}, p_{2}, \ldots, p_{m}\right) ; \sum_{i=1}^{m} p_{i}=1$, where $p_{i}$ is the probability of $\theta_{i}$ being the true state. If the questionnaire $Q$ defined on $\Theta$ is calid and uses $n_{i d}$ questions of resolution d to determine the $i$-th state then (16) holds uith equality iff $p_{i}$ 's are of the form (17). Moreover there exists a heterogeneous questionnaire which satisfies the inequality:

$$
H_{\alpha}(P) \leqq E_{P}^{\alpha} C(Q)<H_{\alpha}(P)+1 ; \quad \alpha>0 .
$$

Proof: For $\alpha=1$, the inequality reduces to (4) which has been proved by Duncan [2]. Hence, we prove only for $\alpha>0, \alpha \neq 1$.

From (17) it is clear that the random charge of a heterogeneous questionnaire satisfies the inequality:

$$
\log _{2} \frac{1}{p_{i}} \leqq \sum_{d=2}^{m} n_{i d} \log _{2} d<\log _{2} \frac{1}{p_{i}}+1
$$

Now, simple computations on (21) give:

$$
1 \leqq \sum_{i=1}^{m} p_{i}^{\alpha} \prod_{d=2}^{m} d^{n_{i d}(\alpha-1)}<2^{\alpha-1}
$$

If we divide (22) by $\sum_{i=1}^{m} p_{i}^{\alpha}$ and take logarithms then on dividing the result so obtained by $(\alpha-1)$ we would immediately get the inequality (20).

We now prove the coding theorem for heterogeneous questionnaires.

THEOREM 4.2: Let there be a state space $\Theta=\left\{\theta_{1}, \theta_{2}, \ldots, \theta_{m}\right\}$ where $\theta_{i}$ 's $(i=1,2, \ldots, m)$ are independent of each other with probability distribution $P=\left(p_{1}, p_{2}, \ldots, p_{m}\right) ; \sum_{i=1}^{m} p_{i}=1, p_{i}>0(i=1,2, \ldots, m)$. If $Q$ be a questionnaire defined on $\Theta$ which determines sufficiently long $M$-sequences of elements of state space, then it is possible to make $E_{P}^{(\alpha, M)} C(Q)$, the average charge of order $\propto$ per state as close to $H_{\alpha}(P)$ as desired.

Proof: Let $s=\left(\theta_{1}, \theta_{2}, \ldots, \theta_{M}\right)$ be a sequence of length $M$ of the elements of $\Theta$ such that the probability of $s$ is:

$$
P(s)=p_{i_{1}} p_{i_{2}} \ldots p_{i_{M}}
$$

R.A.I.R.O. Recherche opérationnelle/Operations Research 
if $\theta_{1}=x_{i_{1}}, \theta_{2}=x_{i_{2}}, \ldots, \theta_{M}=x_{i_{M}}$. If $n_{d}(s)$ be the number of questions of resolution $d$ required to determine the sequence $s$ of length $M$, then:

$$
E_{P}^{(\alpha, M)} C(Q)=\frac{1}{\alpha-1} \log _{2}\left[\frac{\sum_{s}\{P(s)\}^{\alpha} \prod_{d=1}^{\infty} d^{(\alpha-1) n_{d}(s)}}{\sum_{s}\{P(s)\}^{\alpha}}\right] ; \quad \alpha>0, \quad \alpha \neq 1 .
$$

As $\alpha$ entropy is additive, therefore entropy of order $\alpha$ of this product space is:

where:

$$
H_{\alpha, M}\left(P^{*}\right)=M H_{\alpha}(P),
$$

$$
P^{*}=\sum_{s}[P(s)]^{\alpha}
$$

Let $n_{d}(s)$ be the integer satisfying the condition:

$$
-\log _{2} P(s) \leqq \sum_{d=1}^{\infty} n_{d}(s) \log _{2} d<-\log _{2} P(s)+1 .
$$

After simple manipulations and using (24) we get:

$$
\mathrm{H}_{\alpha}(P) \leqq \frac{E_{P}^{(\alpha, M)} C(Q)}{M}<H_{\alpha}(P)+\frac{1}{M} ; \quad \alpha>0, \quad \alpha \neq 1 .
$$

If $\alpha=1$, it is just the ordinary coding theorem.

\section{ACKNOWLEDGMENTS}

The author is grateful to Dr. Prem Nath for some useful discussions.

\section{REFERENCES}

1. J. Aczél, Détermination of All Additive Quasi-Arithmetic Mean Code Word Lengths, Z. Wahr. Verw. Geb., Vol. 29, 1974, pp. 351-360.

2. G. T. Duncan, Heterogeneous Questionnaire Theory, S.I.A.M. J. Appl. Mathematics, Vol. 27, 1974, pp. 59-77.

3. P. NATH, On a Coding Theorem Connected with Rényi's Entropy, Information and Control, Vol. 29, No. 3, 1975, pp. 234-242.

4. A. RÉNYI, On Measures of Entropy and Information, Proc. Fourth Berk. Symp. Math. Stat. Prob., Vol. 1, 1961, pp. 547-561.

5. B. D. ShARMA and A. GARG, Characterization and Lower Bounds for Additive Charges for Heterogeneous Questionnaires, R.A.I.R.O., Vol. 13, No. 4, 1979, pp. 383-393. 